\title{
STATIONARY CONNECTED CURVES IN HILBERT SPACES
}

\author{
Raed Hatamleh, Ahmad Qazza and Hatim Migdadi \\ Department of Mathematics, Jadara University, Irbid-Jordan
}

Received 2014-03-06; Revised 2014-04-16; Accepted 2014-05-17

\begin{abstract}
In this article the structure of non-stationary curves which are stationary connected in Hilbert space is studied using triangular models of non-self-adjoint operator. The concept of evolutionary representability plays here an important role. It is proved that if one of two curves in Hilbert space is evolutionary representable and the curves are stationary connected, then another curve is evolutionary representable too. These curves are studied firstly. The structure of a cross-correlation function in the case when operator, defining the evolutionary representation, has one-dimensional non-Hermitian subspace (the spectrum is discreet and situated in the upper complex half-plane or has infinite multiplicity at zero (Volterra operator)) is studied.
\end{abstract}

Keywords: Stationary Connectedness, Infinitesimal Correlation Matrix, Triangular Operator Model, Channel Operator Element

\section{INTRODUCTION}

It is well known (Rozanov, 1967; Hannan, 2009; Pugachev and Sinitsyn, 2001) that if two stationary random processes of the second order $\xi_{1}(t)$ and $\xi_{2}(t)$ (in what follows we consider that $\left.M \xi_{\alpha}(t)=0\right)$ are stationary connected, then in the corresponding space $H_{\xi}\left(H_{\xi}=\bar{\vee} \underset{k, a}{C_{k, \alpha} \xi_{\alpha}}\left(t_{k}\right), \alpha=1,2 ; k=\overline{1, \infty} ; \overline{\left\{t_{k}\right\}}=[0, \infty)\right.$ is the value space of random processes) they correspond to the stationary curves of the form $\xi_{\alpha}(t)=U_{t} \xi_{0 \alpha}$, where $U_{t}$ is a one-parameter group of unitary operators which always can be represented as $U_{t}=e^{i t A}$, where $A$ is a self-adjoint and, in general, unbounded operator in $H_{\xi}$. If $\xi_{\alpha}(t)(\alpha=1,2)$ are nonstationary random processes, then the question concerning stationary connectedness, i.e., the cross-correlation function dependence upon difference $t-s$, is still opened. The solution of problem may be found in the framework of the Hilbert approach to the construction of the correlation theory of random processes. We will restrict our consideration to the case when corresponding curves in $H_{\xi}$ are evolutionary (linearly) representable (Pugachev and Sinitsyn, 2001; Livshits and Yantsevich, 1979), i.e., may be expressed as $\xi_{\alpha}(t)=e^{i t A_{\alpha}} \xi_{0 \alpha}(\alpha=1,2)$, where $A_{\alpha}$ are linear bounded operators in $H_{\xi}$. For simplicity, we assume that $H_{\xi}=H_{1}=H_{2}, H_{\alpha}$ are Hilbert spaces $H_{\alpha}=\bar{\vee} \bar{C}_{k, \alpha} \xi_{\alpha}\left(t_{k}\right)$.

\section{EVOLUTIONARY REPRESENTABLE STATIONARY CONNECTED CURVES IN $H_{\xi}$}

Let us introduce an infinitesimal correlation matrix (Pugachev and Sinitsyn, 2001) with components:

$$
W_{\alpha \beta}(t, s)=-(\partial t+\partial s) K_{\alpha \beta}(t, s)
$$

For evolutionary representable curves one can easily derive the following expression:

$$
W_{\alpha \beta}(t, s)=\left\langle\frac{A_{\alpha}-A_{\beta}^{*}}{i} \xi_{\alpha}(t), \xi_{\beta}(s)\right\rangle_{H_{\xi}}
$$

Corresponding Author: Raed Hatamleh, Department of Mathematics, Jadara University, Irbid-Jordan 


\section{Lemma 1.}

In order for two random processes to be stationary connected, it is necessary and sufficient that $K_{12}(t, s)$ satisfies the equation:

$$
(\partial t+\partial s) K_{12}(t, s)=0
$$

The necessity is evident, since, in the case of stationary connected processes, $K_{12}(t, s)$ depends on the difference $t-s$ and, for the proof of sufficiency, the new variables $U=t+s, V=t-s$ should be introduced in Equation 2.

\section{Theorem 1.}

If the random processes are stationary connected and are evolutionary representable, then $A_{2}=A_{1}^{*}$.

\section{Proof.}

Let $\left(K_{\alpha, \beta}(t, s)\right)$ be the correlation matrix for a vector random process $\left(\xi_{1}(t), \xi_{2}(t)\right) \in H_{\xi}$. Then the following representation for $K_{\alpha, \beta}(t, s)$ in $H_{\xi}$ is valid, $K_{\alpha \beta}(t, s)=\left\langle\xi_{\alpha}(t), \xi_{\beta}(s)\right\rangle_{H_{\xi}}$, where $\xi_{1}(t), \xi_{2}(t) H_{\xi}$ are corresponding curves in Hilbert space $H_{\xi}$. Since, by hypothesis, $\xi_{\alpha}(t)$ are evolutionary representable:

$$
K_{\alpha \beta}(t, s)=\left\langle e^{i t A a} \xi_{0 \alpha}, e^{i s A} \xi_{0 \beta}\right\rangle_{H_{\xi}}
$$

In view of the stationary connectedness, $K_{12}(t, s)=0$ (Lemma 1) and:

$$
\left(W_{\alpha \beta}(t, s)\right)=\left(\begin{array}{cc}
W_{11}(t, s) & 0 \\
0 & W_{22}(t, s)
\end{array}\right)
$$

From the other hand:

$$
W_{12}(t, s)=\left\langle\frac{A_{1}-A_{2}^{*}}{i} \xi_{1}(t), \xi_{2}(s)\right\rangle_{H_{\xi}}
$$

Hence, it follows from the stationary connectedness that:

$$
\left\langle\left(A_{1}-A_{2}^{*}\right) \sum_{j=1}^{n} a_{j} \xi_{1}\left(t_{j}\right), \sum_{l=1}^{m} b_{l} \xi_{2}\left(t_{l}\right)\right\rangle_{H_{\xi}}=0
$$

i.e.:

$$
\left\langle\left(A_{1}-A_{2}^{*}\right) h_{1}, h_{2}\right\rangle_{H_{\xi}}=0
$$

where, $h_{1}, h_{2}$ are elements of linear span $L=\underset{k, a}{\vee} C_{k, \alpha} \xi_{k, \alpha}\left(t_{k}\right)$. Proceeding to a closure of $L$, we derive that $A_{1}=A_{2}^{*}$ or $A_{2}=A_{1}^{*}$.

Hence, if $\xi_{1}(t)$ and $\xi_{2}(t)$ are evolutionary representable and stationary connected, then in $H_{\xi}$ they are expressed as:

$$
\xi_{1}(t)=e^{i t A} \xi_{01}, \xi_{2}(t)=e^{i t A^{*}} \xi_{02},
$$

and the matrix $\left(W_{\alpha \beta}(t, s)\right)$ is given by:

$W_{\alpha \beta}(t, s)=$

$\left(\begin{array}{cc}\left\langle 2 \operatorname{Im} A \xi_{1}(t), \xi_{1}(s)\right\rangle_{H_{\xi}} & 0 \\ 0 & -\left\langle 2 \operatorname{Im} A \xi_{2}(t), \xi_{2}(s)\right\rangle_{H_{\xi}}\end{array}\right)$.

For the stationary and stationary connected curves $A_{1}=A_{2}=A=A^{*}$, we arrive to the well-known representation of the stationary vector curve in $H_{\xi}: \xi_{\alpha}(t)=e^{i t A} \xi_{0 \alpha}(\alpha=1,2)$ (Rozanov, 1967).

In the subsequent discussion, we restrict our consideration to the most interesting case for application, when the subspace $\overline{\operatorname{Im} A H_{\xi}}$ is finite-dimensional and let $r$ be the subspace dimension.

If the nonstationarity rank of the vector curve $\left(\xi_{1}(t), \xi_{2}(t)\right)$ in $H_{\xi}$ is defined as maximal rank of quadratic forms:

$$
\Phi[z]=\sum_{k, l=1}^{N_{1}, N_{2}}\left\langle\hat{W}\left(t_{k}, t_{l}\right) \overrightarrow{z_{k}}, \overrightarrow{z_{l}}\right\rangle_{E_{2}}, \overrightarrow{z_{k}}=\left(\begin{array}{c}
z_{k}^{(1)} \\
z_{k}^{(2)}
\end{array}\right)
$$

$z_{k}^{(1,2)}$ are complex numbers, then, in the case of $\operatorname{dim} \overline{\operatorname{Im} A H}=r$, one may show that the nonstationarity rank does not exceed $2 r$.

Really, if the operator $A$ is included in the operator complex (Livshits and Yantsevich, 1979; Zolotarev, 2003), $K=\left(A, H_{\xi}, g_{1}, \cdots, g_{r},\left(J_{I m}\right)\right)$, where $\left(J_{I m}\right)$ is an involutive 
matrix $\left(J_{l m}=\overline{J_{l m}}\right.$ and $\left.\sum_{p=1}^{r} J_{i p} J_{p m}=\delta_{i m}\right)$, then we obtain the following expression for $W_{\alpha \beta}(t, s)$ :

$W_{\alpha \beta}(t, s)=\left(\begin{array}{cc}\sum_{l, m=1}^{r} \varphi_{l} J_{l m} \overline{\varphi_{m}(S)} & 0 \\ 0 & -\sum_{l, m=1}^{r} \psi_{l} J_{l m} \overline{\psi_{m}(S)}\end{array}\right)$

where:

$\varphi_{l}(t)=\left\langle e^{i t A} \xi_{01}, g_{l}\right\rangle_{H_{\xi}}, \psi_{l}(t)=\left\langle e^{i t A^{*}} \xi_{02}, g_{l}\right\rangle_{H_{\xi}}$

Then we have:

$$
\Phi[z]=\sum_{l, m=1}^{r} J_{l m} u_{l} \overline{u_{\beta}}-\sum_{l, m=1}^{r} J_{l m} v_{l} \overline{v_{\beta}}
$$

where:

$$
u_{l}=\sum_{k=1}^{N_{1}} \phi_{l}\left(t_{k}\right) z_{k}^{(1)}, v_{l}=\sum_{j=1}^{N_{2}} \psi_{l}\left(t_{j}\right) z_{j}^{(2)}
$$

hence it follows that the rank of $\Phi[z]$ does not exceed the doubled rank of matrix $\left(J_{\mathrm{Im}}\right)$, i.e., $2 r$.

\section{Theorem 2.}

If two curves in $H_{\xi}$ are stationary connected and one of them is evolutionary representable, then another curve is also evolutionary representable.

\section{Proof.}

Let $\xi_{1}(t)$ be evolutionary representable curve, i.e., $\xi_{1}(t)=e^{i t A} \xi_{01}$. In view of stationary connectedness:

$$
W_{12}(t, s)=-(\partial t+\partial s) K_{12}(t, s)=0
$$

But:

$$
\begin{aligned}
& (\partial t+\partial s) K(t, s)=(\partial t+\partial s)\left\langle\xi_{1}(t), \xi_{2}(s)\right\rangle_{H_{\xi}} \\
& =\left\langle\frac{d \xi_{1}(t)}{d t}, \xi_{2}(s)\right\rangle_{H_{\xi}}+\left\langle\xi_{1}(t), \frac{d \xi_{2}(s)}{d s}\right\rangle_{H_{\xi}}
\end{aligned}
$$

$$
\begin{aligned}
& =\left\langle i A \xi(t), \xi_{2}(s)\right\rangle_{H_{\xi}}+\left\langle\xi_{1}(t), \frac{d \xi_{2}(s)}{d s}\right\rangle_{H_{\xi}} \\
& =\left\langle\xi_{1}(t),-i A^{*} \xi_{2}(s)+\frac{d \xi_{2}(s)}{d s}\right\rangle_{H_{\xi}}
\end{aligned}
$$

Hence, it follows (compare with the proof of Theorem 1) that:

$$
\left\langle h,-i A^{*} \xi_{2}(s)+\frac{d \xi_{2}(s)}{d s}\right\rangle_{H_{\xi}}=0, \forall h \in H_{\xi}
$$

i.e.:

$$
-\mathrm{iA} * \xi_{2}(\mathrm{~s})+\frac{\mathrm{d} \xi_{2}(\mathrm{~s})}{\mathrm{ds}}=0 \text { or } \xi_{2}(\mathrm{~s})=\mathrm{e}^{\mathrm{it} \mathrm{A}^{*}} \xi_{02}
$$

\section{THE STRUCTURE OF $W_{12}(t, s)$ AND}

$$
\begin{array}{r}
K_{12}(t, s) \text { FOR STATIONARY } \\
\text { CONNECTED CURVES IN } H_{\xi}
\end{array}
$$

Now we proceed to the investigation of $\left(W_{\alpha \beta}(t, s)\right)$ for the dissipative operator $A . J_{\mathrm{Im}}=\delta_{\mathrm{Im}}$ and:

$$
\left(\mathrm{W}_{\alpha \beta}(\mathrm{t}, \mathrm{s})\right)=\left(\begin{array}{cc}
\sum_{\mathrm{l}=1}^{\mathrm{r}} \varphi_{1}(\mathrm{t}) \overline{\varphi_{1}(\mathrm{~s})} & 0 \\
0 & -\sum_{\mathrm{l}=1}^{\mathrm{r}} \psi_{1}(\mathrm{t}) \overline{\psi_{1}(\mathrm{~s})}
\end{array}\right) .
$$

If the dissipative operator $A$ has discreet spectrum:

$$
\sigma(A)=\left\{\alpha_{k}+i \frac{\beta_{k}^{2}}{2}\right\}_{k=1}^{\infty}, \text { with } \sum_{k=1}^{\infty} \beta_{k}^{2}<\infty
$$

then, if $r=1$, the functions $\varphi(t)$ and $\psi(t)$ (index $l$ is omitted) may be restored only by spectrum and initial values $\xi_{01}$ and $\xi_{02}$.

\section{Theorem 3.}

Let:

$$
\left\{\lambda_{\mathrm{k}}=\alpha_{\mathrm{k}}+\mathrm{i} \frac{\beta_{\mathrm{k}}^{2}}{2}\right\}, \mathrm{k}=\overline{1, \infty}
$$


be arbitrary complex numbers in upper complex half plane and:

$$
\sum_{\mathrm{k}=1}^{\infty} \beta_{\mathrm{k}}^{2}<\infty
$$

and $a_{1}, a_{2}, \cdots, b_{1}, b_{2}, \cdots$ be arbitrary sequences with:

$$
\sum_{\mathrm{k}=1}^{\infty}\left|\mathrm{a}_{\mathrm{k}}\right|^{2}<\infty \text { and } \sum_{\mathrm{k}=1}^{\infty}\left|\mathrm{b}_{\mathrm{k}}\right|^{2}<\infty
$$

Then there exists the evolutionary representable Gauss vector process with matrix Equation 6 and 7:

$$
\mathrm{W}_{\alpha \beta}(\mathrm{t}, \mathrm{s})=\left(\begin{array}{cc}
\varphi(\mathrm{t}) \overline{\varphi(\mathrm{s})} & 0 \\
0 & \psi(\mathrm{t}) \overline{\psi(\mathrm{s})}
\end{array}\right)
$$

where:

$$
\varphi(t)=\sum_{k=1} \alpha_{k} \Lambda_{k}(t), \psi(t)=\sum_{k=1} b_{k} \Lambda_{k}^{*}(t)
$$

with:

$$
\begin{aligned}
& \Lambda_{k}(t)=\overline{-\frac{1}{2 \pi i} \int_{r} e^{-i t \lambda} \frac{\sqrt{2 \operatorname{Im} \lambda_{k}}}{\overline{\lambda_{k}}-\lambda} \prod_{j=1}^{k-1} \frac{\lambda_{j}-\lambda}{\overline{\lambda_{j}}-\lambda} d \lambda} \\
& \Lambda_{k}^{*}(t)=-\overline{\frac{1}{2 \pi i} \int_{r} e^{-i t \lambda} \frac{\sqrt{2 \operatorname{Im} \lambda_{k}}}{\lambda_{k}-\lambda} \prod_{j=k+1}^{\infty} \frac{\overline{\lambda_{j}}-\lambda}{\lambda_{j}-\lambda} d \lambda .}
\end{aligned}
$$

\section{Proof.}

Let us build a triangular model of operator $\hat{A}$ acting in the space $\ell^{2}$ with the help of the set of $\lambda_{1}, \lambda_{2}, \cdots$ (Livshits and Yantsevich, 1979; Zolotarev, 2003). Then consider in $\ell^{2}$ the model curves:

$$
\xi_{1}(t)=e^{i \hat{A} \hat{A}} \xi_{01}, \xi_{2}(t)=e^{i \hat{A}^{*}} \xi_{02}
$$

where:

$$
\xi_{01}=\left(\begin{array}{c}
\alpha_{1} \\
\alpha_{2} \\
\vdots
\end{array}\right), \xi_{02}=\left(\begin{array}{c}
b_{1} \\
b_{2} \\
\vdots
\end{array}\right)
$$

Taking into account the expressions (5) for $\varphi(t)$ and $\psi(t)$, with $A=\hat{A}$ and the expressions for $e^{i \hat{A}}$ and $e^{i \hat{A}^{*}}$ via the corresponding resolvents:

$$
e^{i t B}=-\frac{1}{2 \pi i} \oint_{\gamma} e^{-i t \lambda}(B-\lambda I)^{-1} d \lambda,\|B\|<\infty
$$

the contour $\gamma$ encloses the spectrum of operator $B$ (Pugachev and Sinitsyn, 2001), we obtain the expressions (6) and (7).

The existence of Gauss vector processes results from the possibility of the correlation matrix $\left(K_{\alpha \beta}(t, s)\right)$ to be restored by the scalar products calculations:

$$
K_{\alpha \beta}(t, s)=\left\langle e^{i \hat{A} \hat{A}} \xi_{0 \alpha}, e^{i s \hat{A} *} \xi_{0 \beta}\right\rangle_{H_{\xi}}
$$

The Gauss complex valued vector process $\left(\xi_{1}(t), \xi_{2}(t)\right)$ may be reconstructed, but not uniquely defined, by the correlation matrix (Hannan, 2009).

In our case, the most interesting is the structure:

$$
K_{12}(t, s)=\left\langle e^{i(t-s) \hat{A}} \xi_{01}, \xi_{02}\right\rangle_{H_{\xi}}
$$

First we consider a case when:

$$
\xi_{02}=\left(\begin{array}{c}
b_{1} \\
b_{2} \\
\vdots
\end{array}\right)=\hat{g}
$$

where, $\hat{g}$ is a channel element of dissipative operator A with discrete spectrum. Then using results from chapter V (Livshits and Yantsevich, 1979) we derive the expression:

$$
K_{12}(t-s)=\sum_{l=1}^{\infty} \alpha_{l} \Lambda_{l}(t-s)
$$

where, $\Lambda_{l}(t)$ are special $\Lambda$-functions (Livshits and Yantsevich, 1979) built only by the operator spectrum $\left\{\lambda_{k}\right\}$. For example, if all $\lambda_{k}$ are different, then:

$$
\Lambda_{l}(t)=\sum_{j=1}^{\infty} d_{l, j} e^{i t \lambda_{j}}
$$

If $\xi_{02}=\hat{\mathrm{g}}$, then we can derive similar expression (8) for $K_{12}(t-s)$ using formula: 


$$
e^{-i(t-s) \hat{A}^{*}} \xi_{02}=-\frac{1}{2 \pi i} \oint_{\gamma} e^{-i(t-s) \lambda}(\hat{A} *-\lambda I)^{-1} \xi_{02} d \lambda .
$$

The calculation of resolvent for the triangular model operator $\hat{A}^{*}$ on $\xi_{02} \neq \hat{g}$ is reduced to the solution of inhomogeneous difference equation:

$$
\mathrm{y}_{\mathrm{k}+1}-\mathrm{y}_{\mathrm{k}} \cdot \frac{\lambda_{\mathrm{k}}-\lambda}{\lambda_{\mathrm{k}}}-\lambda=\gamma_{\mathrm{k}+1}-\gamma_{\mathrm{k}}
$$

Where:

$$
\begin{aligned}
& \gamma_{k}=\frac{b_{k}}{\beta_{k}}, y_{k}=\frac{\overline{\lambda_{k}}-\lambda}{\beta_{k}} f_{k}(\lambda), \\
& f_{k}(\lambda)=\left((\hat{A} *-\lambda I)^{-1} \xi_{02}\right)_{k}
\end{aligned}
$$

Solving Equation (9) for $f_{k}(\lambda)$, we obtain Equation 10:

$$
f_{k}(\lambda)=\frac{\beta_{k}}{\overline{\lambda_{k}}-\lambda}\left(\prod_{j=1}^{k-1} \frac{\lambda_{j}-\lambda}{\overline{\lambda_{j}}-\lambda}+\sum_{j=1}^{k-2} \prod_{j=1}^{k-2}\left(\prod_{i=1}^{k-j} \frac{\lambda_{k-l}-\lambda}{\overline{\lambda_{k-l}}-\lambda}\right) \gamma_{j+1}\right),
$$

$k \geq 2$

$$
f_{1}(\lambda)=\frac{b_{1}}{\overline{\lambda_{1}}-\lambda}
$$

If $\left(\xi_{02}\right)$ coincides with channel element $g$ of the operator $A$, then all $\gamma_{k}=1\left(b_{k}=\beta_{k}\right)$ and (10) changes to the expression obtained in the reference (Pugachev and Sinitsyn, 2001).

Taking into consideration (10), for $\Lambda_{k}(t)$ we obtain:

$$
\Lambda_{k}(t)=\overline{-\frac{1}{2 \pi i} \oint_{\gamma} e^{-i t \lambda} f_{k}(\lambda) d \lambda}
$$

Thus:

$$
\begin{aligned}
& \Lambda_{1}(t)=\bar{b}_{1} e^{i t \lambda_{1}} \\
& \Lambda_{2}(t)=\frac{i \bar{b}_{1} \beta_{1} \beta_{2}}{\lambda_{1}-\lambda_{2}} e^{i t \lambda_{1}}+\left(\bar{b}_{2}-\frac{i \bar{b}_{1} \beta_{1} \beta_{2}}{\lambda_{1}-\lambda_{2}}\right) e^{i t \lambda_{2}},\left(\lambda_{1} \neq \lambda_{2}\right)
\end{aligned}
$$

and, in general, if all $\left\{\lambda_{k}\right\}$ are different, then:

$$
\Lambda_{k}(t)=\sum_{j=1}^{k} c_{k, j} e^{i t \lambda_{j}}
$$

If we use as a model operator the Volterra operator:

$$
\hat{A f}=i \int_{0}^{x} f(u) d u
$$

acting in a space $L_{[0, l]}^{2}$, then one can easily obtain the following expressions for $\varphi(t)$ and $\psi(t)$ :

$$
\begin{aligned}
& \varphi(t)=\int_{0}^{1} a(x) J_{0}(2 \sqrt{t u}) d u \\
& \psi(t)=\int_{0}^{1} b(x) J_{0}(2 \sqrt{t(1-u)}) d u
\end{aligned}
$$

where, $J_{0}$ is the Bessel function of a zero order.

Using the universal models of dissipative operators (Livshits and Yantsevich, 1979; Zolotarev, 2003), one may also consider any case of $2 \leq r<\infty$.

\section{CONCLUSION}

Spectral representation for non stationary curves, which are stationary connected, in the case when the operator $A$ satisfies the requirements of completeness, i.e., it has full chain of decreasing subspaces $H=H_{0} \supset H_{1} \supset \cdots$ with $H_{k-1} \Theta H_{k}=1$, may be obtained using the theory of open systems associated with the operator colligations containing the operators $A$ or $A^{*}$ (Livshits and Yantsevich, 1979; Zolotarev, 2003).

\section{REFERENCES}

Hannan, E.J., 2009. Multiple Time Series. 1st Edn., Join Wiley and Sons, Inc., New York, ISBN-10: 0470317132, pp: 536.

Livshits, M.S. and A.A. Yantsevich, 1979. Operator Colligations in Hilbert Spaces. 1st Edn., V.H. Winston, Washington. pp: 212.

Pugachev, V.S. and I.N. Sinitsyn, 2001. Stachastic Systems: Theory and Application. 1st Edn., Singapore, World Scientific., pp: 684.

Rozanov, Y.A., 1967. Stationary Random Processes. 1st Edn., Holden-Day, San Francisco, pp: 211.

Zolotarev, V., 2003. Analytic methods spectral representation of nonselfadjoint and nonunitary operators. Kharkov National University. Kharkov, Russian. 